\title{
Projeto de um Transceptor para Integração em Nanossatélites
}

\author{
Vinícius M. Pereira, Diego P. Fumagalli, Lucas S. Pereira e Marcos V. T. Heckler
}

\begin{abstract}
Resumo-Este artigo apresenta o desenvolvimento e validação experimental de uma topologia de transceptor para integração em nanossatélites com dois canais de comunicação: enlace de subida em $401 \mathrm{MHz}$ e enlace de descida em 2,26 GHz. Analisase, inicialmente, o cenário no qual o sistema completo (nanossátelite com transceptor embarcado) deverá operar. Na sequência, analisam-se os componentes (amplificadores, filtros e mixer) a serem empregados no projeto. Por fim, o protótipo construído e caracterizado experimentalmente é apresentado e discutido.
\end{abstract} link.

Palavras-Chave-Transceptor, Nanossatélite, Uplink, Down-

Abstract-This paper presents the development and experimental validation of a transceiver topology for integration in nano-satellites with two communication channels: uplink at $401 \mathrm{MHz}$ and downlink at $2.26 \mathbf{~ G H z}$. Initially, the scenario of the complete system (nano-satellite with the integrated transceiver) is analyzed. Then, the components (amplifiers, filters and mixer) to be used in the design are analyzed. Finally, the experimental characterization of the prototype is presented and discussed.

Keywords - Transceiver, Nanosatellite, Uplink, Downlink.

\section{INTRODUÇÃo}

O Sistema Brasileiro de Coleta de Dados Ambientais (SBCDA) consiste em uma rede de coleta de dados formada por satélites e Plataformas de Coleta de Dados (PCDs), distribuídas ao longo do território nacional e de sua costa marítima. Cada PCD possui sensores embarcados, específicos para aplicações como: monitoramento hidrográfico, previsão de fenômenos climáticos, estudo de correntes oceânicas e eventuais alterações químicas atmosféricas, e observação de níveis de poluição. Os dados coletados são periodicamente atualizados e armazenados no hardware da plataforma e, a cada intervalo de aproximadamente 200 segundos, são enviados para o espaço por um transmissor operando na frequência de $401,62 \mathrm{MHz}$ [1].

Até meados do ano 2017, o SBCDA possuía os satélites SCD1 e SCD2, lançados nos anos de 1993 e 1998, respectivamente. Sua órbita é circular, com inclinação de $25^{\circ} \mathrm{em}$ relação à linha do Equador e a $750 \mathrm{~km}$ de altitude. A cada

Vinícius M. Pereira, Grupo de Óptica, Micro e Nanofabricação de Dispositivos - GOMNDI, Universidade Federal do Pampa - Unipampa, AlegreteRS, e-mail: viniciusmp2.aluno@unipampa.edu.br; Diego P. Fumagalli, Laboratório de Eletromagnetismo, Micro-ondas e Antenas - LEMA, Universidade Federal do Pampa - Unipampa, Alegrete-RS, e-mail: diegofumagalli@unipampa.edu.br; Lucas S. Pereira, Laboratório de Eletromagnetismo, Micro-ondas e Antenas - LEMA, Universidade Federal do Pampa - Unipampa Alegrete-RS; Marcos V. T. Heckler, Laboratório de Eletromagnetismo, Microondas e Antenas - LEMA, Universidade Federal do Pampa - Unipampa, Alegrete-RS, e-mail: marcosheckler@unipampa.edu.br. Este trabalho foi parcialmente financiado pelo Programa de Bolsas de Desenvolvimento Acadêmico (PDA) Unipampa. passagem sobre as regiões das PCDs, os dados coletados são recebidos e, em sequência, retransmitidos, na frequência de 2267,52 MHz, para as estações terrenas, localizadas nas cidades de Cuiabá (MT) e Alcântara (MA). Estas, são responsáveis pelo rastreio dos satélites, recepção e encaminhamento dos dados para o Centro de Controle de Missão (CCM), situado em Natal (RN). No CCM, as informações são tratadas e armazenadas para acesso e consulta dos usuários finais do sistema [1].

Em [2], propôs-se a substituição dos satélites atuais por nanossatélites. Os enlaces de comunicação entre PCDs e nanossatélites (uplink), e nanossatélites e Centro de Missão e Coleta de Dados - CMCD (downlink) serão implementados por quatro sistemas independentes. O canal de uplink deve operar na frequência de $401 \mathrm{MHz}$. O canal de downlink deve operar em 2,26 GHz. O sistema de telemetria, comando e controle (TC\&C) deve operar em $145 \mathrm{MHz}$. O quarto enlace será dedicado ao sistema de telecomando, operando em $435 \mathrm{MHz}$. Em [3], apresentaram-se os requisitos técnicos de uma proposta de nanossatélite para o projeto CONASAT - Constelação de Nanossatélites Ambientais, visando ao desenvolvimento de um protótipo com massa total de 1 a $10 \mathrm{~kg}$. Assim, suas antenas e circuitos eletrônicos devem ser compactos e de mínimo peso.

Em [4], apresentaram-se as especificações de dimensionamento físico, carga útil, subsistemas de energia e de armazenamento e processamento eletrônico de dados, de um nanossatélite $1 U$ em desenvolvimento, denominado CONASAT0. Sua estrutura é de baixo custo e reduzidas dimensões físicas. Em [5], o autor apresentou o desenvolvimento da primeira versão do sistema de controle de bordo do "cubesat" CONASAT-0, que consiste em um hardware digital que contempla os subsistemas de telemetria, telecomando, comunicação e energia.

Em [6], uma topologia de transceptor a ser embarcada em um modelo de engenharia de nanossatélite foi proposta. Esta, divide-se em: frontend em $401 \mathrm{MHz}$, para a recepção do sinal de uplink, e backend em 2,26 GHz, para retransmissão dos dados para a Terra. Neste estudo, considerou-se que, inicialmente, os testes e demonstrações de funcionamento do nanossatélite sejam desenvolvidos em terra, considerando uma distância de aproximadamente 100 metros entre a fonte transmissora e o protótipo. Em [7], um modelo de engenharia de nanossatélite, cujas redes de antenas de uplink e downlink operam em $401 \mathrm{MHz}$ e 2,26 GHz, respectivamente, foi apresentado. Seu objetivo é validar o princípio de funcionamento, em termos eletromagnéticos, do enlace formado entre PCDs e nanossatélite (uplink), processamento e retransmissão do sinal 
recebido (transceptor embarcado) e do enlace formado por nanossatélite e estação receptora (downlink).

No presente trabalho, apresenta-se a construção e validação prática do protótipo da topologia de transceptor proposta em [6]. O projeto visa à integração ao modelo de engenharia de nanossatélite apresentado em [7]. Nas seguintes seções, serão mostrados o cenário de operação, os procedimentos adotados para o desenvolvimento do transceptor e os resultados obtidos durante a validação de seus blocos individuais e de seu protótipo integrado.

\section{Modelagem do RÁdio EnlaCe}

Por se tratar de um modelo de engenharia para testes e demonstrações terrestres, o cenário para qual o transceptor é dimensionado consiste em um ambiente outdoor, onde a antena transmissora é posicionada afastada cerca de 100 metros do nanossatélite, conforme ilustrado na Figura 1.

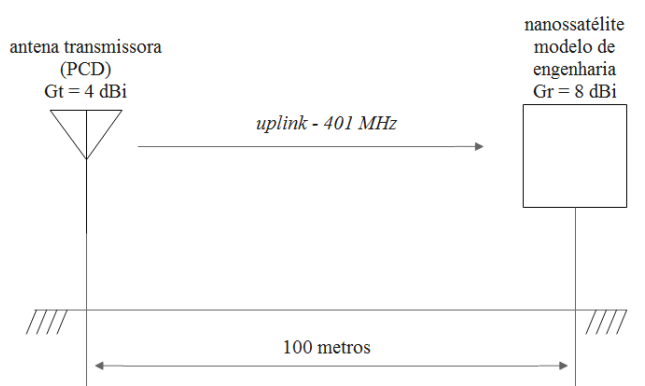

Fig. 1. Ilustração do cenário para testes do nanossatélite. (Fonte: Adaptado de [8]).

Como visto na Figura 1, a antena transmissora (que simula uma PCD) possui ganho de aproximadamente $4 \mathrm{dBi}$ e a rede de antenas receptoras (no nanossatélite), cerca de $8 \mathrm{dBi}$. Considera-se, também, que o sinal transmitido pelo canal de uplink tenha potência de $0 \mathrm{dBm}$ em $401 \mathrm{MHz}$.

Para o cálculo deste enlace, as especificações descritas anteriormente devem ser aplicadas na equação de Friis

$$
P_{r}(\mathrm{dBm})=P_{t}(\mathrm{dBm})+G_{t}(\mathrm{dBi})+G_{r}(\mathrm{dBi})-L_{F S}(\mathrm{~dB}),
$$

para se estimar o nível de potência recebido pelo nanossatélite. $\mathrm{Na}$ equação (1), o termo $L_{F S}$ corresponde à atenuação do espaço livre e pode ser calculado por

$$
L_{F S}(\mathrm{~dB})=20 \log \left(\frac{4 \pi R}{\lambda_{0}}\right),
$$

onde $P_{r}(\mathrm{dBm})$ é a potência recebida em $\mathrm{dBm}, P_{t}(\mathrm{dBm})$ é a potência transmitida em $\mathrm{dBm}, G_{r}(\mathrm{dBi})$ é o ganho da antena receptora em $\mathrm{dBi}, G_{t}(\mathrm{dBi})$ é o ganho da antena transmissora em $\mathrm{dBi}, R$ é a distância entre as antenas em metros e $\lambda_{0}$ é o comprimento de onda no espaço livre [9].

A Tabela I mostra os parâmetros estimados teoricamente no cálculo do enlace de subida e as especificações consideradas. Esses dados permitem estabelecer a topologia de transceptor mais adequada em termos de processamento do sinal recebido e mitigação das perdas do espaço livre $\left(L_{F S}\right)$ devidas ao canal
TABELA I

ParÂMetros estimados do ENLACE DE SUbida. (Fonte: AdAPtado

\begin{tabular}{|c|c|}
\hline Parâmetro & Valor estimado \\
\hline $\begin{array}{ll}f(\mathrm{MHz}) \\
\end{array}$ & 401,00 \\
\hline$P_{t}(\mathrm{dBm})$ & 0,00 \\
\hline$G_{t}(\mathrm{dBi})$ & 4,00 \\
\hline$G_{r}(\mathrm{dBi})$ & 8,00 \\
\hline$R \quad(\mathrm{~m})$ & 100,00 \\
\hline$L_{F S}(\mathrm{~dB})$ & 65,50 \\
\hline$P_{r}(\mathrm{dBm})$ & $-52,50$ \\
\hline
\end{tabular}
DE [6]).

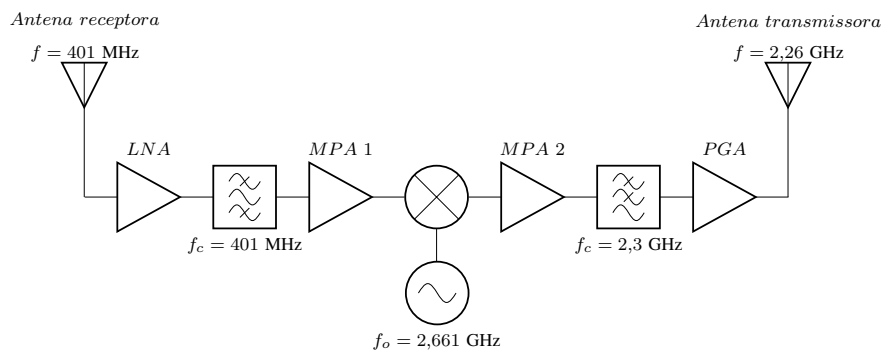

Fig. 2. Diagrama de blocos do transceptor proposto. (Fonte: retirado de [6]).

modelado. O diagrama de blocos resultante é mostrado na Figura 2.

O sistema proposto (Figura 2) pode ser dividido em dois estágios: frontend e backend. O frontend compreende o estágio inicial, formado por um amplificador de baixo ruído (do inglês, low-noise amplifier - LNA), um filtro passa-banda (do inglês, band-pass filter - BPF), um amplificador de média potência (do inglês, medium power amplifier - MPA) e um mixer passivo, para translação de $401 \mathrm{MHz}$ a $2,26 \mathrm{GHz}$. O backend consiste no estágio final do transceptor, em que o sinal é condicionado para sua retransmissão. Assim, utilizam-se, após o mixer, um MPA, um filtro passa-baixa (Low-pass Filter LPF) e um amplificador de potência (do inglês, power gain amplifier - PGA) [10], [11].

\section{RESUltados EXPERIMENTAIS}

\section{A. Validação dos Blocos do Sistema}

Após a definição da topologia para o transceptor (Figura 2), prosseguiu-se com a definição, em termos de modelos comerciais, dos amplificadores e mixer a serem empregados. Os filtros foram calculados e modelados no software $\mathrm{HFSS}^{\circledR}$.

De acordo com os estudos desenvolvidos em [12] e [13], considerou-se a utilização de alguns componentes empregados pelos autores. Assim, para o frontend, especificaram-se os seguintes modelos de componentes: LNA - HMC374; MPA1 - ERA-1SM+; mixer - HMC316MS8. Os parâmetros $S$ dos amplificadores, medidos considerando o intervalo de frequência entre $300 \mathrm{MHz}$ e $500 \mathrm{MHz}$, são mostrados nas Figuras 3 (HMC374) e 4 (ERA-1SM+).

O ganho medido do amplificador "LNA" (parâmetro $S_{21}$, Figura 3), cerca de 15,05 dB, trata-se de um resultado bastante interessante pois, aliado à baixa figura de ruído do dispositivo (cerca de 1,50 dB) garante que o sistema completo apresente, também, baixa figura de ruído e, aliado ao ganho medido do 


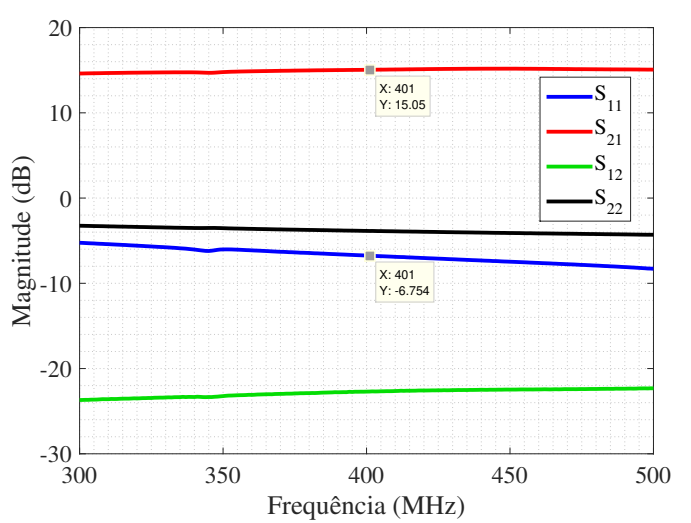

Fig. 3. Parâmetros $S$ medidos LNA: HMC374. (Fonte: Adaptado de [8]).

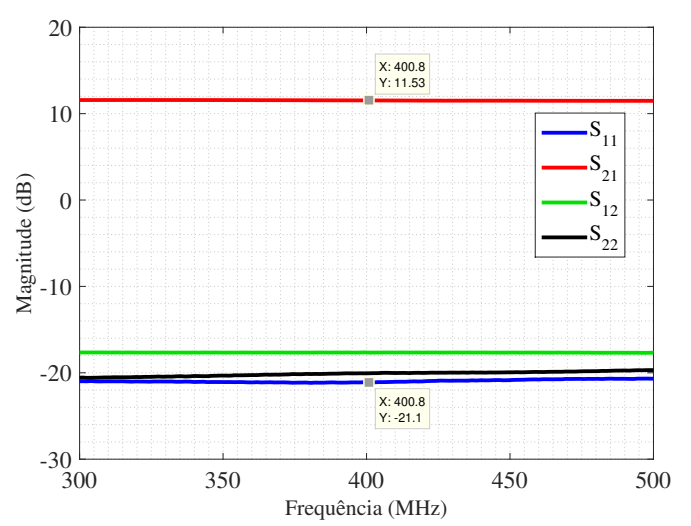

Fig. 4. Parâmetros $S$ medidos MPA1: ERA-1SM+. (Fonte: Adaptado de [8]).

amplificador "MPA1", possibilita que o frontend disponha de um ganho total de aproximadamente 19,24 dB (considerando a perda devido ao mixer passivo).

$\mathrm{O}$ mixer foi validado quanto à translação em frequência do sinal recebido (401 MHz) para sua retransmissão $(2,26 \mathrm{GHz})$. Para isso, fixou-se que o sinal do oscilador local deveria ter frequência de $2,661 \mathrm{GHz}$, de modo que $f_{\text {out }}=2,661-$ $0,401=2,26 \mathrm{GHz}$. A Figura 5 mostra o espectro do sinal de saída do mixer para um sinal de entrada com $0 \mathrm{dBm}$ de potência.

Uma topologia de filtro passa-banda cuja resposta em frequência, em termos de largura de banda passante e rejeição de frequências interferentes $(433 \mathrm{MHz}$ - frequência livre - e $435 \mathrm{MHz}$ - frequência de comunicação do sistema de telecomando), foi proposta em [14]. Tal estudo, contudo, encontrase ainda em fase de desenvolvimento, visando sua futura integração à topologia de transceptor proposta no presente trabalho.

Para o estágio de backend, dois amplificadores comerciais foram empregados, sendo MPA2 o modelo GALI-2+ [13] e PGA o modelo PGA-105+. Projetou-se, também, um filtro passa-baixas de ordem 7 em tecnologia de microfita e topologia stepped impedance, cujo embasamento teórico para seu projeto é descrito em [15].

Os parâmetros $S$ medidos dos amplificadores, considerando o intervalo de frequência entre $2 \mathrm{GHz}$ e $2,5 \mathrm{GHz}$, são mos-

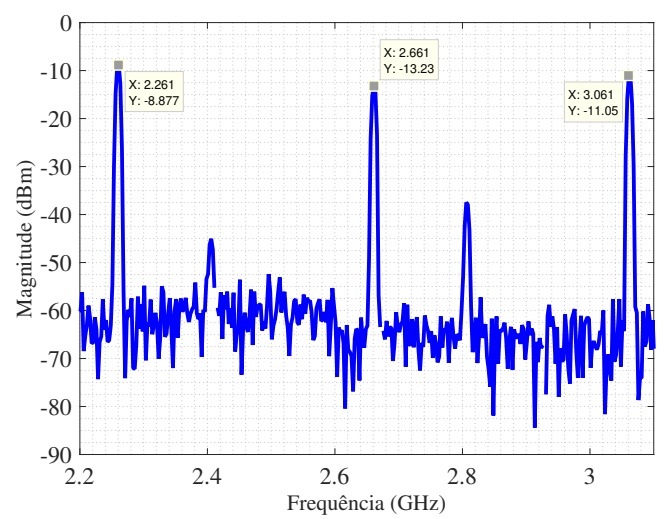

Fig. 5. Espectro medido do sinal de saída do mixer. (Fonte: Adaptado de [8]).

trados nas Figuras 6 (GALI-2+) e 7 (PGA-105+).

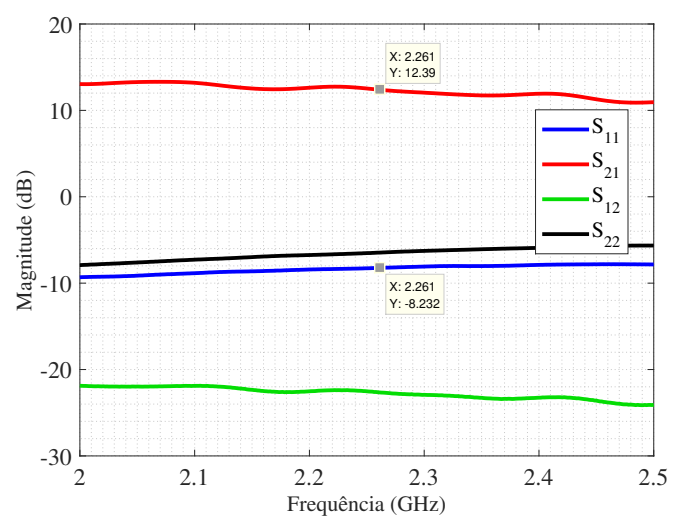

Fig. 6. Parâmetros $S$ medidos MPA2: GALI-2+. (Fonte: Adaptado de [8]).

Os ganhos medidos dos amplificadores "MPA2" e "PGA" (parâmetros $S_{21}$, Figuras 6 e 7) combinados, garantem ao estágio de backend um ganho total de aproximadamente $23,43 \mathrm{~dB}$, considerando a perda de inserção do filtro passabaixa. Sabendo-se que o transceptor deve receber um sinal com baixíssima potência, processá-lo e retransmiti-lo, é necessário que sua implementação seja baseada em amplificadores com ganhos suficientemente altos, como observado nos dispositivos caracterizados nas Figuras 3, 4, 6 e 7.

O protótipo construído e os parâmetros $S$ medidos do filtro passa-baixa são mostrados nas Figuras 8 e 9, respectivamente.

Para o cálculo do ganho total do transceptor e de sua figura ruído (do inglês, noise figure - NF) total, têm-se como base os parâmetros de ganho e/ou perda de inserção de cada componente caracterizado isoladamente, elencados na Tabela II.

A partir dos parâmetros listados na Tabela II, pôde-se estimar o ganho total do transceptor, tal que

$$
G_{\text {total }}(d B)=15,05+11,53-7,34+12,40-2,07+13,10
$$

$$
G_{\text {total }}(d B)=42,67 d B
$$




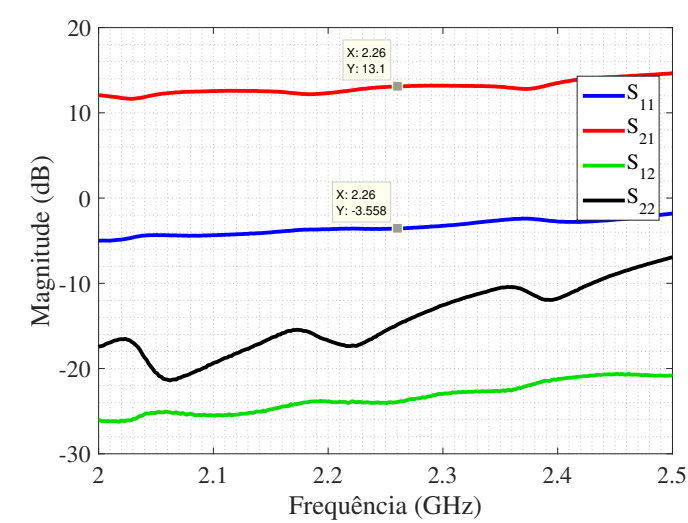

Fig. 7. Parâmetros $S$ medidos PGA: PGA-105+. (Fonte: Adaptado de [8]).

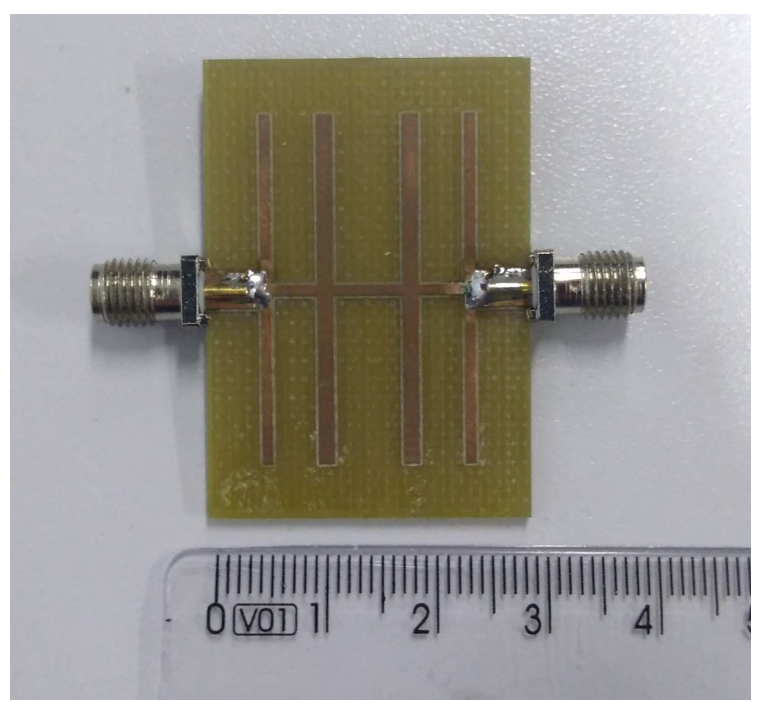

Fig. 8. Protótipo construído: filtro passa-baixa. (Fonte: Retirado de [8]).

A figura de ruído total do sistema foi estimada como aproximadamente 1,5, em valor absoluto, ou 1,76 dB [8].

A topologia de transceptor resultante, implementada e discutida na seguinte seção, possui diagrama de blocos como ilustrado na Figura 10.

\section{B. Validação do Transceptor Integrado}

Como etapa final, construiu-se um protótipo do transceptor completo, cujos componentes foram validados na seção anterior. Na Figura 11, mostra-se o sistema implementado em uma única placa de circuito impresso. Utilizou-se a topologia de guia de ondas coplanares aterrados (do inglês, coplanar waveguide grounded - CPWG), considerando o laminado FR4, $\operatorname{com} \varepsilon_{r}=4,8, \tan \delta=0,02$ e espessura de $1,6 \mathrm{~mm}$. As linhas de transmissão foram dimensionadas com largura $W_{C P W G}=$ $1,7 \mathrm{~mm}$, gap de $0,5 \mathrm{~mm}$ para o plano de terra (GND) superior e vias de aterramento espaçadas entre si e do centro da linha de $2,5 \mathrm{~mm}$, para fins de se garantir que $Z_{L T}=50 \Omega$, além de evitar excitação de propagação de ondas TEM entre placas paralelas.

Para alimentação dos dispositivos ativos (amplificadores), utilizaram-se duas fontes $\mathrm{CC}$, sendo uma configurada com

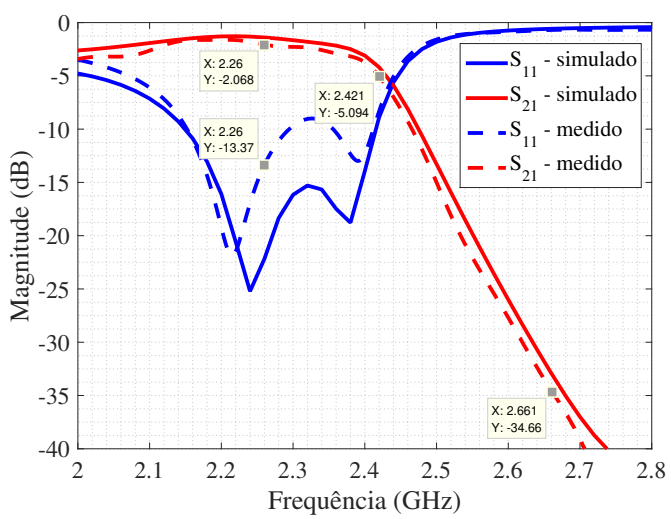

Fig. 9. Parâmetros $S$ simulados e medidos: filtro passa-baixa. (Fonte: Adaptado de [8]).

TABELA II

PARÂMETROS DE GANHO E FIGURA DE RUÍDO DOS BLOCOS DO SISTEMA. (FONTE: ADAPTADO DE [8]).

\begin{tabular}{|c||c|c|c|}
\hline Componente & $f(\mathrm{MHz})$ & $G(\mathrm{~dB})$ & $N F(\mathrm{~dB})$ \\
\hline HMC374 & 401 & 15,05 & 1,50 \\
\hline ERA-1SM+ & 401 & 11,53 & 4,30 \\
\hline HMC316MS8 & $401-2260$ & $-7,34$ & 7,34 \\
\hline GALI-2+ & 2260 & 12,40 & 4,60 \\
\hline LPF & 2260 & $-2,07$ & 2,07 \\
\hline PGA-105+ & 2260 & 13,10 & 1,90 \\
\hline
\end{tabular}

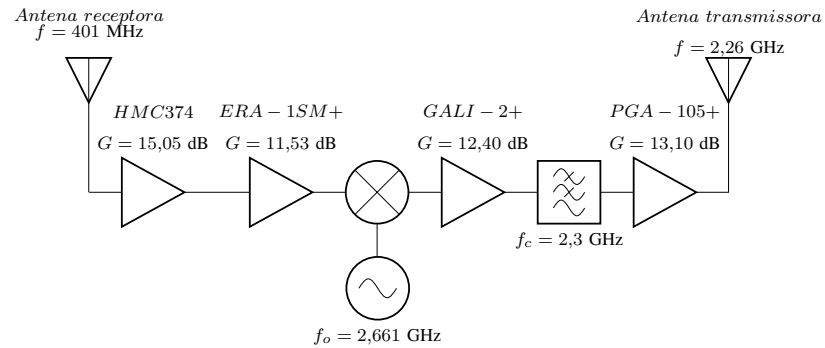

Fig. 10. Diagrama de blocos do transceptor proposto. (Fonte: Adaptado de [8]).

tensão de $+5 \mathrm{~V}$ (para conexão à rede de polarização dos amplificadores HMC374 e PGA-105+) e a segunda com tensão de $+12 \mathrm{~V}$ (para conexão à rede de polarização dos amplificadores ERA-1SM+ e GALI-2+).

$\mathrm{O}$ sinal aplicado à entrada do transceptor foi produzido por um gerador de sinais de RF, com potência de $-30 \mathrm{dBm}$ em $401 \mathrm{MHz}$, conforme se pode observar no espectro da Figura 12. O sinal do oscilador local também foi produzido por um gerador de sinais de RF, com frequência de $2,661 \mathrm{GHz}$ e potência de aproximadamente $+15 \mathrm{dBm}$.

Aplicando-se o sinal de entrada (Figura 12) ao transceptor, mediu-se o espectro do sinal de saída, mostrado na Figura 13. Este sinal resultante possui frequência de $2,26 \mathrm{GHz}$ e mais elevado nível de potência, estando, assim, pronto para ser retransmitido pelo sistema de downlink. Para o caso específico, analisado em laboratório, o protótipo construído apresentou ganho total de aproximadamente $44,40 \mathrm{~dB}$, superando, inclusive, a estimativa inicial de $42,67 \mathrm{~dB}$, calculada na seção 


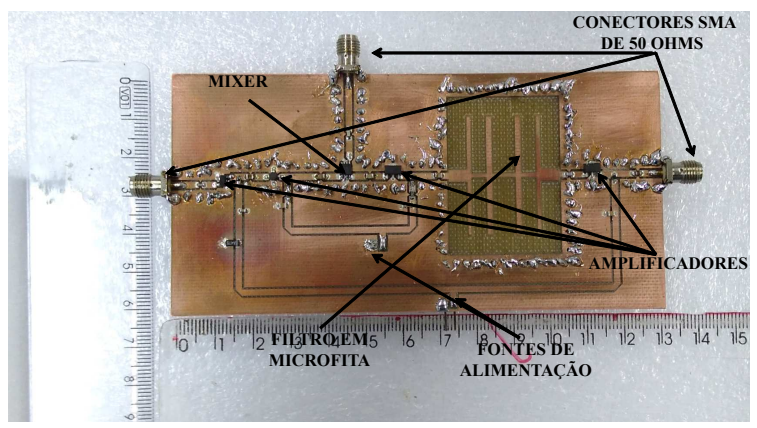

Fig. 11. Protótipo construído: transceptor. (Fonte: Retirado de [8]).

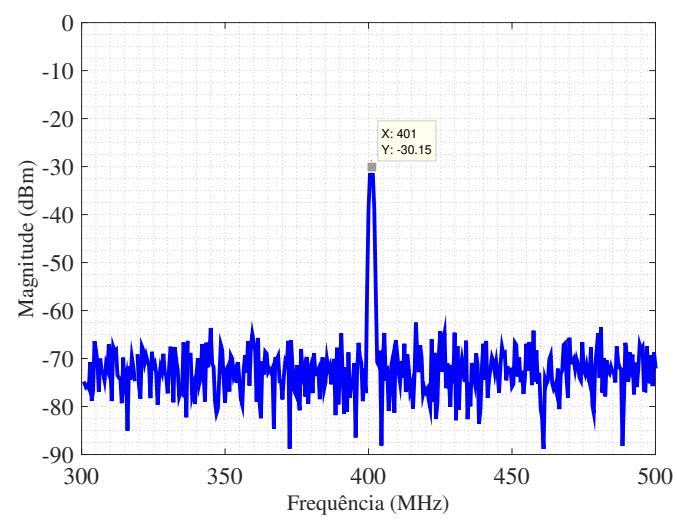

Fig. 12. Espectro do sinal aplicado na entrada do transceptor. (Fonte: Adaptado de [8]).

anterior.

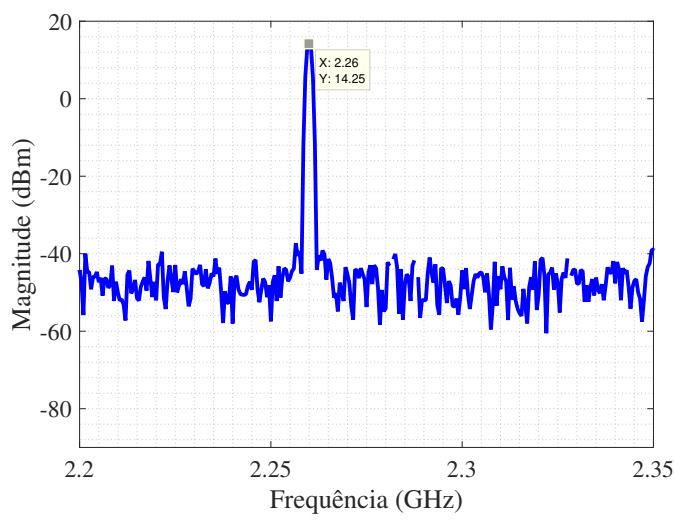

Fig. 13. Espectro do sinal medido na saída do transceptor. (Fonte: Adaptado de [8]).

\section{CONCLUSÕES}

O trabalho desenvolvido apresentou a proposta de uma topologia de transceptor para integração em nanossatélites vinculados ao projeto CONASAT, com frontend operando em $401 \mathrm{MHz}$, e backend em 2,26 GHz. Por meio da construção do protótipo e medições em bancada, averiguou-se que esta atende às especificações de processamento do sinal recebido do canal de uplink, e sua translação em frequência para sua posterior retransmissão no canal de downlink, estabelecidas no item II. Observou-se, também, que o ganho total do sistema obtido com a integração de todos os componentes em uma só placa de circuito impresso $(44,40 \mathrm{~dB})$ foi superior àquele estimado somando-se os ganhos e subtraindo-se as perdas de inserção de cada bloco validado separadamente $(42,67 \mathrm{~dB})$. Este fato pode ser explicado devido à soma das perdas de inserção introduzidas pelos diversos conectores utilizados na caracterização dos componentes separadamente na primeira etapa de validação experimental.

Como etapas futuras de desenvolvimento, os autores avaliam o projeto de um novo filtro passa-faixa, para elevar a isolação do enlace de subida de dados de demais sistemas e fontes de interferência.

\section{AGRADECIMENTOS}

Os autores agradecem à Universidade Federal do Pampa (Unipampa) pela estrutura laboratorial oferecida e pelo auxílio financeiro por meio do Programa de Bolsas de Desenvolvimento Acadêmico (PDA).

\section{REFERÊNCIAS}

[1] J. S. S. Lima, L. S. Jotha, R. B. Biondi, P. S. de Aquino e V. P. da Silva Neto. "Documento de Requisitos Preliminares - Fase A (DRP). Natal, 2012”. Disponível em $<$ http://www.inpe.br/crn/conasat/arquivos/Documentos/gerais/CONASATDocumentodeRequisitosPreliminares.pdf $>$. Acesso em: 19 mar. 2021.

[2] M. J. M. De Carvalho, J. S. S. Lima, L. S. Jotha e P. S. Aquino. "CONASAT - Constelação de Nanossatélites para Coleta de Dados Ambientais,” em XVI Simpósio Brasileiro de Sensoriamento Remoto, Foz do Iguaçu-PR, Abr. 2013, pp. 9108-9115. 2013.

[3] K. I. P. Queiroz, S. M. Dias, J. M. L. Duarte e M. J. M. de Carvalho. "Uma Solução para o Sistema Brasileiro de Coleta de Dados Ambientais Baseada em Nanossatélites," em Holos, Dez. 2018, pp. 132-142, DOI: 10.15628/holos.2018.6307.

[4] A. C. R. Alves, S. M. Dias, K. I. P. de M. Queiroz, M. J. M. de Carvalho, J. M. L. Duarte. "CONASAT-0: Visão Geral do Nanossatélite Desenvolvido," em II Congresso Aeroespacial Brasileiro - CAB2019, Santa Maria-RS, Set. 2019, pp. 1-9. DOI: 10.29327/2cab2019.224823.

[5] A. C. R. Alves. "Controle de Bordo para Nanossatélites: Desenvolvimento e Aplicação ao Projeto CONASAT”. Dissertação de Mestrado. Universidade Federal do Rio Grande do Norte - UFRN. Natal-RN, 2019.

[6] V. M. Pereira, J. M. Vieira, L. S. Pereira e M. V. T. Heckler. "Projeto de um Circuito Transceptor de Frequências para Integração em Nanossatélites," em XXXV SIMPÓSIO BRASILEIRO DE TELECOMUNICAÇÕES E PROCESSAMENTO DE SINAIS - SBrT2017, São Pedro-SP, Set. 2017, pp. 1-5. DOI:10.14209/sbrt.2017.139.

[7] J. M. Vieira, E. Yoshimoto, F. G. Ferreira, V. M. Pereira, and M. V. T. Heckler. "UHF and S-band Antenna Arrays for Nano-satelite-based Data-relay," in 12th European Conference on Antennas and Propagation (EuCAP 2018), Abr. 2018, pp. 1-5, DOI: 10.1049/cp.2018.0984.

[8] V. M. Pereira. "Projeto de um Circuito Transceptor de Frequências para Integração em Nanossatélites". Trabalho de Conclusão de Curso. Universidade Federal do Pampa - Unipampa. Alegrete-RS, 2017.

[9] J. A. J. Ribeiro. Propagação das Ondas Eletromagnéticas - Princípios e Aplicações. 2.ed. São Paulo: Erica, 2008.

[10] L. E. Frenzel. Fundamentos de Comunicações Eletrônicas: Linhas, Micro-ondas e Antenas. Porto Alegre: AMGH, 2013.

[11] C. W. Sayre. Complete Wireless Design. 2.ed. Mc Graw Hill, 2008.

[12] V. L. Barbosa. "Estudo e Desenvolvimento de uma Rede Linear de Antenas de Microfita com Feixe Chaveado e de um Receptor Homódino para Aplicação em Banda ISM”. Dissertação de Mestrado. Universidade Federal de Santa Maria - UFSM. Santa Maria-RS, 2016.

[13] M. P. Magalhães. "Design and Analysis of an Antenna Array System for Communication Using High-Altitude Plataforms". Master thesis. Universidade Federal do Pampa - Unipampa. Alegrete-RS, 2017.

[14] V. Pereira e M. V. T. Heckler. "Miniaturização de Filtros em Tecnologia de Microfita para Aplicações em Banda UHF," em Anais do Salão Internacional de Ensino, Pesquisa e Extensão, v. 9, n. 2, 2017.

[15] D. M. Pozar. Microwave Engineering. 3.ed. John Wiley \& Sons, 2005. 